\title{
Noninvasive Neurophysiological Monitoring in Acute Ischemic Stroke Treatment
}

\author{
Moreira Joana ${ }^{1}$, Mota Cardoso Carolina ${ }^{2}$, Godinho Liliane ${ }^{3}$ and Traila Campos Manuel ${ }^{1}$ \\ ${ }^{1}$ Department of Anestesiology, Critical Care \& Emergency Medicine, Portugal \\ ${ }^{2}$ Department of Anestesiology, Centro Hospitalar Tâmega e Sousa, Portugal \\ ${ }^{3}$ Department of Anestesiology, Centro Hospitalar e Universitário de Coimbra, Portugal
}

Submission: March 08, 2017; Published: May 31, 2017

*Corresponding author: Manuel Traila Campos, Department of Anesthesiology, Critical Care \& Emergency Medicine, Centro Hospitalar do Porto, Largo Professor Abel Salazar, 4099-001 Porto, Portugal; Tel: +351 963901117; Email: blycampos@gmail.com

Abstract

Introduction: Acute brain ischemia treatment aims to rapid re canalize occluded brain vessels. Currently, the treatment outcome is measured by clinical and imagiological scales. During endovascular therapy with conscious sedation, neurological clinical examination can be performed. For patients under general anaesthesia only angiography can monitor outcome. Although non-invasive neurophysiological monitoring during stroke treatment has not yet been recommended by any randomized clinical studies, some small studies have already highlighted its importance.

Discussion: It was found a correlation between rSO2 measured with NIRS and angiographic classification. Although NIRS seems to be a good tool for monitoring the viability of brain tissue, it has some limitations. EEG requires an expert interpretation, however quantitative techniques obviate this. QEEG methods have demonstrated improved detection and localization capabilities that reliably correlate with the clinical examination and lesion location on neuroimaging. TCD has a high efficiency in detecting occlusions in thrombolysis-eligible patients and is also useful in prognostication of stroke. It isn't a good intraprocedure option due to its important limitations. Bioimpedance is also a decent tool for stroke diagnostic and treatment monitoring. The considerable percentage of false-negatives is a limitation for its use.

Conclusion: NIRS and qEEG used as intraprocedure monitoring for acute ischemic stroke have shown clinical benefits. Despite the promising results observed, there is a need for more clinical studies in order to validate the benefits of this novel strategy.

Keywords: Acute ischemic stroke; Neurophysiological monitoring; NIRS; qEEG; General anesthesia; Thrombectomy; Thrombolysis

Abbreviations: BSI: Brain Symmetry Index; CT: Computed Tomography; DAR: Delta/Alpha Ratio DTABR: (Delta + Theta)/(Alpha + Beta) Power Ratio; NHISS: National Institutes of Health Stroke Scale; EEG: Electro Encephalogram; MCA: Middle Cerebral Artery; NIRS: Near InfraRed Spectroscopy; OR: Odds Ratio; PI: Perturbative Index; r-tPA: Plasminogen Activator; rSO2: Regional Oxygen Saturation; TICI: Thrombolysis in Cerebral Infarction; TCD: Transcranial Doppler

\section{Introduction}

Acute brain ischemia treatment aims to rapid recanalyze occluded brain vessels to ameliorate regional reperfusion and salvage of the threatened tissues. The outcome is measured by clinical scales: National Institutes of Health Stroke Scale (NHISS) [1] and modified Rankin scale [2]; imagiological studies like brain computed tomography (CT) [3]; and recanalization rates by angiography (Thrombolysis In Cerebral Infarction-TICI) [4,5].

In the AHA/ASA Guidelines for the Early Management of Patients With Acute Ischemic Stroke, the rapid administration of intravenous recombinant tissue-type plasminogen activator (r-tPA) to appropriate patients remains the mainstay of early treatment, even if endovascular treatments are being considered, with improvement of functional outcome at 3 to 6 months when given within 4.5 hours of clinical onset (Class I; Level of Evidence A). Endovascular therapy with a stent retriever in eligible patients is also a class I, level of Evidence A since 2015 guidelines update [6].

The Society for Neuroscience and Anesthesiology and Critical Care provided information for the best practice in perioperative management of patients with acute ischemic stroke undergoing endovascular therapy. As for neurophysiological monitoring during conscious sedation, they regarded focused neurological clinical examination as standard and correlated with volume of brain salvaged. As for patients under general anesthesia there 
isn't currently compelling evidence to recommend advanced neuro monitoring outside the angiographic information [7]. Nevertheless, case-studies and case-reports have already highlighted that intraprocedural monitoring can contribute to minimize clinical impact of intraoperative cerebral injury [8].

\section{Discussion}

\section{Brain oxygenation}

Assessing recanalization and reperfusion in acute ischemic stroke management is a major issue of morphological imaging methods such as angiographic/perfusion CT or multimodal MRI. However, they do not provide continuous monitoring of the tissue oxygen consumption. Noninvasive cerebral oxygenation monitoring with Near Infra-Red Spectroscopy (NIRS) allows a real time monitorization of regional oxygen saturation (rSO2) of the brain. This approach measures the saturation of brain oxygen through the ratio of Oxy- and Deoxy-hemoglobin, by placing a skin sensor on the frontal region. NIRS is already used to detect brain ischemia in carotid endarterectomy, craniectomycerebral arteriovenous malformations resection and brain tumour resections [8].

Hametner et al. [9] conducted an observational study in 43 patients elected for endovascular therapy for acute ischemic stroke caused by anterior cerebral circulation occlusion, in whom they monitored NIRS before, during and after the intervention performed under general anesthesia. The area under the curve $10 \%$ below baseline of rSO2 values correlated with reperfusion angiographic classification (TICI) and was suggested as an independent negative predictor of successful reperfusion (odds ratio(OR) 0,998, 95\% CI, p=0,023). Variability measure of rSO2 after revascularization was associated with poor functional outcome (modified Rankin scale) (OR 0,06, 95\% $\mathrm{CI}, \mathrm{p}=0,046)$. rSO2 inter hemispheric difference lower values of end-intervention was proposed as an independent predictor of death at day 90 (OR 0,144, 95\% CI, p=0,008). Also, rSO2 was correlated with mean arterial blood pressure variability, that supports NIRS as adjunctive tool to guide blood pressure management during and possibly post intervention. 9

Thomas Ritzenthaler et al. [10] monitored recanalization and reperfusion during mechanicalthrombectomy after large middle cerebral artery stroke. A clear correlation between recanalization, improvement of $\mathrm{rSO} 2$ and decreasing in the rSO2differential was documented in case of successful thrombectomy. Conversely, when clot removal procedure failed, no change in rSO2-diff was observed [10].

Although NIRS seems to be a good tool for monitoring the viability of brain tissue, there are some limitations [11], and the current set-up may require an improved stroke-specific technological adjustment (e.g., more optodes in particular positions, simultaneous perfusion assessment) in future studies [9].
At present, there is not sufficient evidence to support NIRS use as a surrogate or replacement for any invasive monitoring modality. However, the technology is improving rapidly with introductions of novel methods of data acquisition and analysis. These exciting developments have the potential to enhance the utility of NIRS as a noninvasive neurological monitoring tool that can provide information that is as useful to the clinician as are currently available invasive modalities [12].

\section{Quantitative Electroencephalography Techniques}

Electroencephalogram (EEG) reflects extracellular currents mainly resulting from the summated responses of excitatory and inhibitory postsynaptic currents of cortical pyramidal cells and the transcortical and subcortical influences that modulate these potencials. Typical of ischemic stroke is the rapid appearance of high-amplitude, slow activity in the delta frequency range $(1-3 \mathrm{~Hz})$. This detection sensivity is superior to current imaging methods and to the clinical examination if the patient is asleep, sedated, paralyzed or has altered consciousness. At intermediate levels of ischemia we can see attenuation of beta $(14-30 \mathrm{~Hz})$ followed by slowing of alpha $(8-13 \mathrm{~Hz})$. EEG abnormalities arise when the normal cerebral blood flow of $50-70 \mathrm{ml} / 100 \mathrm{~g} / \mathrm{min}$ declines to $25-30 \mathrm{ml} / 100 \mathrm{~g} / \mathrm{min}$ [13]. This is still a technique that requires an expert interpretation and is not easily available in most centers. Quantitative techniques obviate this. Quantitative (QEEG) measures of specific EEG features are likewise valuable in brain ischemia. In addition, compared with qualitative, visual analysis of EEG alone, QEEG methods have demonstrated improved detection and localization capabilities, that reliably correlate with lesion location on neuroimaging. Reductions in delta power, DAR (Delta/alpha ratio), DTABR ((delta + theta)/(alpha + beta) power ratio) or BSI (brain symmetry index) during the acute ischemic stroke interval are generally correlated with concomitant NHISS and with relatively better functional outcomes (Modified Rankin scale) at up to 12 months post-stroke [14].

There have been some reported outcomes from EEG/qEEG bedside monitor in the immediate response to thrombolytic therapy. Reports have been showing reduction in global delta power within 10-25min following commencement of intravenous tPA and preceding symptomatic changes by $1-3.5 \mathrm{~h}$. This pre-treatment delta was generated by ischemic cortical tissue which was re-perfused post-thrombolysis [14]. DAR was found to be the most accurate index for discriminating between radiologically-confirmed acute ischemic stroke and age-matched controls without brain ischemia which may prove valuable for promptly detecting cerebral ischemia and inform decisions regarding reperfusion therapies [15].

Sheikh et al. [16] reported 2 cases were QEEG was useful for expedite decision regarding intra-arterial clot retrieval in acute ischemic stroke. Patient A (74 year woman) was admitted with NIHSS score of 15 and CT Perfusion showed a infarct core 
in the left frontal premotor region. DAR was above 12 prior to intravenous alteplase administration and remained highly abnormal thereafter, accurately indicating lack of successful therapy (discharge NIHSS score 11; moderate severe disability at 3 months). Patient B (74 year woman) was admitted with NIHSS score of 19 and CTPerfusion showed penumbra involving approximately one third of the rightmiddle cerebral artery (MCA) territory. DAR remained abnormal ( $>7.5$ ) and symptoms severe (NIHSS 19) following intravenous alteplase, but soon after ensuing intra-arterial clot retrieval DAR had dropped below 3.7, accurately indicating successful reperfusion (mild symptoms at discharge; NIHSS 2) [16].

Schleiger et al. [17] analyzed 20 patients in the post ischemic stroke period $(62-101 \mathrm{~h}$ after onset of symptoms of MCA) comparing QEEG indices from the standard 19 versus 4 frontal electrodes and correlating these with cognition-specific, functional outcome measures. A highly significant correlation was obtained between frontal, DAR assessed at approximately $72 \mathrm{~h}$ post-stroke onset and cognitive outcome measures assessed at mean 3.5 months post-stroke. This correlation was stronger than the non-significant correlation between cognitive outcomes and global DAR (computed from all nineteen electrodes) [17].

\section{Transcranial Doppler}

Transcranial Doppler (TCD) sonography is a non-invasive, inexpensive, bed-side and safe technique that uses a pulsed Doppler transducer for assessment of intracerebral blood flow. TCD has been reported to have a sensitivity of $80 \%$ and a specificity of $90 \%$ compared with cerebral angiography in patients with MCA stroke [18].

Alexandrov et al. [19] have shown that TCD has the highest efficiency in detecting occlusions in thrombolysis-eligible patients (69\% of these patients). If urgent TCD is normal, there is almost an $89 \%$ chance that subsequent angiography will not discover an arterial occlusion. Moreover, in $26 \%$ of all patients TCD provided additional information to angiography, helping to refine the severity of stenosis, to provide clues to stroke pathogenesis and to show whether recanalisation has occurred following intravenous thrombolysis [19].

TCD is also useful in prognostication of stroke. A normal TCD at $6 \mathrm{~h}$ post-ischaemic stroke is an independent predictor of early improvement [20]; TCD may predict occurrence of spontaneous haemorrhagic transformation in ischaemic infarcts [21]; in acute MCA stroke, blood flow velocity on TCD of $<30 \mathrm{~cm} / \mathrm{s}$ within $12 \mathrm{~h}$ after stroke correlated with poor recovery [22]; and TCD has a role in the detection of microembolic signals, which help in stratification of risk of recurrence of stroke or transient ischaemic attack [23]. Although its established diagnostic value, it isn't a current intra procedure practice due to its limitations: operator dependent; transtemporal window can be absent in up to $5-20 \%$ of the patients, so the vessels cannot be properly insonated due to inadequacy of the acoustic windows [19]; the probe may interfere with the angiographic image; and diagnostic criteria for arteries other than the MCA are lacking.

\section{Bioimpedance}

The principle of bioimpedance is based onthe particular value of conductivity of each tissue, being possible to distinguish them when an electrical current is applied. The results can be converted in the perturbative index (PI).

Liu et al. [24] verified there was no significant difference between the PI of right and left hemispheres in healthy volunteers, when bioimpedance was applied to the brain tissue. However, non-normal matter (e.g. hematoma or edema) can lead to the change of cerebral electrical impedance. In this study, $81.8 \%$ (27) of patients with ischemic stroke had a higher numeric data of PI in the infarct side and there was a positive correlation with the volume of infarction. The larger the volume of infarct, the larger the edema, consequently the PI value was higher, and the outcome was worse. Being a safe, continuous, non-invasive, cost-effective and bed side monitor, it may be a sensitive parameter for monitoring the change of brain edema and may be useful as a complement to CT or MRI scan for monitoring treatment or determining neurological outcome. Nevertheless, in this study there was a considerable percentage of false-negatives. The authors hypothesize being due to its low sensitivity if the focal lesions are located in the midline or near the midline; if the volume of lesions is less than $15-20 \mathrm{ml}$; and in multiple lacunar infarction [24]. This results were confirmed in subsequent studies developed by the same department [25-27].

\section{Conclusion}

Currently, there isn't sufficient evidence to support the use of any neurophysiological monitor technique, mentioned in this review, during acute ischemic stroke treatment under general anesthesia. Transcranial Doppler and bioimpedance have shown diagnostic and follow-up value in acute ischemic stroke patients, however their intraprocedure use has too many limitations. NIRS and qEEG used as intraprocedure monitoring have shown clinical benefits. Although only a few and small observational studies support their value, they seem promising. Despite the promising results observed, there is a need for more clinical studies in order to validate the benefits of this novel strategy.

\section{References}

1. https://stroke.nih.gov/resources/scale.htm.

2. Bruno A, Shah N, Akinwuntan AE, Close B, Switzer JA (2013) Stroke Size Correlates with Functional Outcome on the Simplified Modified Rankin Scale Questionnaire. J Stroke Cerebrovasc Dis 22(6): 781-783.

3. Birenbaum D, Bancroft LW, Felsberg GJ (2011) Imaging in acute stroke. West J Emerg Med 12(1): 67-76.

4. Mueller HS, Dyer A, Greenberg MA (1985) The Thrombolysis in Myocardial Infarction (TIMI) trial. Phase I findings. TIMI Study Group. N Engl J Med 312(14): 932-936.

5. Rha J-H, Saver JL (2007) The impact of recanalization on ischemic stroke outcome: a meta-analysis. Stroke 38(3): 967-973. 


\section{Open Access Journal of Neurology \& Neurosurgery}

6. Powers WJ, Derdeyn CP, Biller J, Coffey CS, Hoh BL, et al. (2015) 2015 American Heart Association/American Stroke Association Focused Update of the 2013 Guidelines for the Early Management of Patients With Acute Ischemic Stroke Regarding Endovascular Treatment. Stroke 46(10): 3020-3035.

7. Dhakal LP, Díaz-Gómez JL, Freeman WD (2015) Role of Anesthesia for Endovascular Treatment of Ischemic Stroke. Stroke 46(6): 1748-1754.

8. Badenes R, Gruenbaum SE, Bilotta F (2015) Cerebral protection during neurosurgery and stroke. Curr Opin Anaesthesiol 28(5): 532-536.

9. Hametner C, Stanarcevic P, Stampfl S, Rohde S, Veltkamp R, et al (2015) Noninvasive Cerebral Oximetry during Endovascular Therapy for Acute Ischemic Stroke: An Observational Study. J Cereb Blood Flow Metab 35(11): 1722-1728.

10. Ritzenthaler T, Cho T-H, Luis D, Berthezene $\mathrm{Y}$, Nighoghossian $\mathrm{N}$ (2015) Usefulness of near-infrared spectroscopy in thrombectomy monitoring. J Clin Monit Comput 29(5): 585-589.

11. Ferrari M, Mottola L, Quaresima V (2004) Principles, Techniques, and Limitations of Near Infrared Spectroscopy. Can J Appl Physiol 29(4): 463-487.

12. Davies DJ, Su Z, Clancy MT, Lucas SJ, Dehghani H, et al. (2015) NearInfrared Spectroscopy in the Monitoring of Adult Traumatic Brain Injury: A Review. J Neurotrauma 32(13): 933-941.

13. Jordan KG (2004) Emergency EEG and continuous EEG monitoring in acute ischemic stroke. J Clin Neurophysiol 21(5): 341-352.

14. Finnigan S, van Putten MJAM (2013) EEG in ischaemic stroke: Quantitative EEG can uniquely inform (sub-)acute prognoses and clinical management. Clin Neurophysiol 124(1): 10-19.

15. Finnigan S, Wong A, Read S (2016) Defining abnormal slow EEG activity in acute ischaemic stroke: Delta/alpha ratio as an optimal QEEG index. Clin Neurophysiol 127(2): 1452-1459.

16. Sheikh N, Wong A, Read S, Coulthard A, Finnigan S (2013) QEEG may uniquely inform and expedite decisions regarding intra-arterial clot retrieval in acute stroke. Clin Neurophysiol 124(9): 1913-1914.

17. Schleiger E, Sheikh N, Rowland T, Wong A, Read S, et al. (2014) Frontal
EEG delta/alpha ratio and screening for post-stroke cognitive deficits: The power of four electrodes. Int J Psychophysiol 94(1): 19-24.

18. Camerlingo M, Casto L, Censori B, Ferraro B, Gazzaniga GC, et al. (1993) Transcranial Doppler in acute ischemic stroke of the middle cerebral artery territories. Acta Neurol Scand 88(2): 108-111.

19. Alexandrov AV, Black SE, Ehrlich LE, Caldwell CB, Norris JW (1997) Predictors of hemorrhagic transformation occurring spontaneously and on anticoagulants in patients with acute ischemic stroke. Stroke 28(6): 1198-1202.

20. Babikian VL, Feldmann E, Wechsler LR, Newell DW, Gomez CR, et al. (2000) Transcranial Doppler Ultrasonography: Year 2000 Update. J Neuroimaging 10(2): 101-115.

21. Toni D, Fiorelli M, Zanette EM, Sacchetti ML, Salerno A, et al. (1998) Early spontaneous improvement and deterioration of ischemic stroke patients. A serial study with transcranial Doppler ultrasonography. Stroke 29(6): 1144-1148.

22. Halsey JH (1988) Prognosis of acute hemiplegia estimated by transcranial Doppler ultrasonography. Stroke 19(5): 648-649.

23. Valton L, Larrue V, le Traon AP, Massabuau P, Géraud G (1998) Microembolic signals and risk of early recurrence in patients with stroke or transient ischemic attack. Stroke 29(10): 2125-2128.

24. Liu LX, Dong WW, Wang J, Wu Q He W, et al. (2005) The role of noninvasive monitoring of cerebral electrical impedance in stroke. Acta Neurochir Suppl 95: 137-140.

25. Liu L, Dong W, Ji X, Chen L, Chen L, et al. (2006) A new method of noninvasive brain-edema monitoring in stroke: cerebral electrical impedance measurement. Neurol Res 28(1): 31-37.

26. He LY, Wang J, Luo Y, Dong WW, Liu LX (2010) Application of noninvasive cerebral electrical impedance measurement on brain edema in patients with cerebral infarction. Neurol Res 32(7): 770-774.

27. Lou JH, Wang J, Liu LX, He LY, Yang H, et al. (2012) Measurement of brain edema by noninvasive cerebral electrical impedance in patients with massive hemispheric cerebral infarction. Eur Neurol 68(6): 350357.

\section{Your next submission with Juniper Publishers} will reach you the below assets

- Quality Editorial service

- Swift Peer Review

- Reprints availability

- E-prints Service

- Manuscript Podcast for convenient understanding

- Global attainment for your research

- Manuscript accessibility in different formats

( Pdf, E-pub, Full Text, Audio)

- Unceasing customer service

Track the below URL for one-step submission

https://juniperpublishers.com/online-submission.php 